\title{
Praktische Fragen der Inanspruchnahme von Freizügigkeit*
}

\author{
Stephan Fasshauer
}

A. Vorbemerkungen 235

B. Anmerkungen zur quantitativen Bedeutung der Fragestellung 237

C. Anmerkungen zu den Rechtsanwendungen 239

D. Zentrale Fragestellungen aus Sicht der Praxis 242

I. Fragen im Rahmen der Erwerbsphase $\quad 242$

II. Fragen im Rahmen des Rentenbezugs 246

1. Vorbemerkungen 246

$\begin{array}{ll}\text { 2. Berechtigung auf Leistungen nach dem deutsch-polnischen } & \\ \text { Sozialversicherungsabkommen von } 1975 \text { (DPSVA 1975) } & 247\end{array}$

3. Berechtigung auf Leistungen nach dem Fremdrentengesetz (FRG) 248

4. Weitere Entwicklung $\quad 249$

III. Beitreibung von Forderungen 250

IV. Elektronischer Datenaustausch 251

1. Überblick 251

2. Electronic Exchange of Social Security Information (EESSI) 252

3. Europäisches Online-Auskunftsverfahren mit Polen (EOA-Polen) 253

4. Sterbedatenabgleich Deutschland-Polen 254

E. Fazit 255

\section{A. Vorbemerkungen}

Seit Gründung der Europäischen Union bzw. Europäischen Gemeinschaft vor mehr als fünf Jahrzehnten erlebte diese in verschiedenen Etappen eine erhebliche Erweiterung. Nunmehr bilden 27 Staaten mit fast einer halben Milliarde Menschen die Europäische Union. Alle Länder vereint das Ziel, einen gemeinsamen europäischen Binnenmarkt zu bilden und damit die Voraussetzungen dafür zu schaffen, dass für Personen, Waren, Dienstleistungen und Kapital dieselbe Freizügigkeit zwischen den Mitgliedstaaten besteht wie innerhalb der einzelnen Länder. Das bedeutet, dass nicht nur grenzüber-

* Der Verfasser dankt Frau Seidel und Herrn Scheewe von der Deutschen Rentenversicherung BerlinBrandenburg für ihre wertvolle Unterstützung bei der Erstellung des Manuskriptes. 
schreitend gereist und gewohnt, sondern beispielsweise auch gearbeitet und studiert wird. 1

Diese Freizügigkeit, wie sie im täglichen Leben bereits vielfach erlebt wird, basiert auf dem Grundrecht auf Freizügigkeit nach Art. 45 der Charta der Grundrechte der Europäischen Union (GRC) ${ }^{2}$ sowie auf der Grundfreiheit der Arbeitnehmerfreizügigkeit nach Art. 45 ff. des Vertrags über die Arbeitsweise der Europäischen Union (AEUV). Gewährleistet wird dieses Grundrecht durch das Unionssrecht und hierbei für den Bereich der gesetzlichen Rentenversicherung durch die Verordnungen (EG bzw. EWG) zur Koordinierung der Systeme der sozialen Sicherheit. Nachteile, die aus der Inanspruchnahme der Freizügigkeit entstehen könnten, sollen durch diese vermieden bzw. reduziert werden.

Das europäische Unionsrecht regelt insoweit mit Blick auf die Sicherung im Alter im Wesentlichen: ${ }^{3}$

- Die Gleichbehandlung der Staatsangehörigen aller EU/EWR-Staaten und der Drittstaatsangehörigen mit rechtmäßigem Wohnsitz in der EU,

- die Zusammenrechnung der nach den jeweiligen nationalen Rechtsvorschriften der EU/EWR-Staaten zurückgelegten Versicherungszeiten für den Erwerb des Leistungsanspruches sowie für die Berechnung der Leistung,

- die Gleichstellung der Staatsgebiete der EU/EWR-Staaten für den Export der Leistungen.

In der Praxis resultieren hieraus für die Betroffenen verschiedene Problemfelder, die im Weiteren bezogen auf Polen aus Sicht der gesetzlichen Rentenversicherung aufgegriffen werden. Die Bedeutung der Fragestellungen nimmt dabei in Anzahl und Komplexität beständig zu. So sind die Fallzahlen im Verhältnis von Deutschland zu Polen seit dem EU-Beitritt zum 01.05.2004 und seit der Öffnung des deutschen Arbeitsmarktes zum 01.05.2011 stark angestiegen. Dies zeigen die Auswertungen aller Träger der gesetzlichen Rentenversicherung in Deutschland, die sich aufgrund der festgelegten Zuständigkeiten für diese Fälle im Wesentlichen verantwortlich zeigen (Deutsche Rentenversicherung (DRV) Bund, die DRV Knappschaft-Bahn-See und die DRV BerlinBrandenburg). ${ }^{4}$

1 Vgl. umfassend hierzu: www.europa.eu.

2 Vgl. hierzu u. a.: Voet van Vormizeele, in: Schwarze/Becker/Hatje/Schoo (Hrsg.), EU-Kommentar, 2. Auflage 2009, Art. 45 GRC, Rn. 1 ff.

3 Vgl. zu den Verordnungen mit weiteren Quellen u. a.: Eichenhofer, Europarecht, in: Eichenhofer/Rische/Schmähl (Hrsg.), Handbuch der gesetzlichen Rentenversicherung SGB VI, 2011, S. 1049 ff.

4 Die DRV Berlin-Brandenburg ist auf Regionalebene die Verbindungsstelle zu Polen. 


\section{B. Anmerkungen zur quantitativen Bedeutung der Fragestellung}

Nach dem aktuellen Migrationsbericht der Bundesregierung erfolgten im Berichtsjahr 2009 mit jeweils rund 123.000 die meisten $\mathrm{Zu}$ - bzw. Fortzüge nach bzw. aus Deutschland im Verhältnis zu Polen. ${ }^{5}$ Dabei stand die temporäre Arbeitsmigration eindeutig im Vordergrund (z.B. Saisonarbeitskräfte).

Abbildung 1: Zu- und Fortzüge nach den häufigsten Herkunfts- und Zielländern 2009

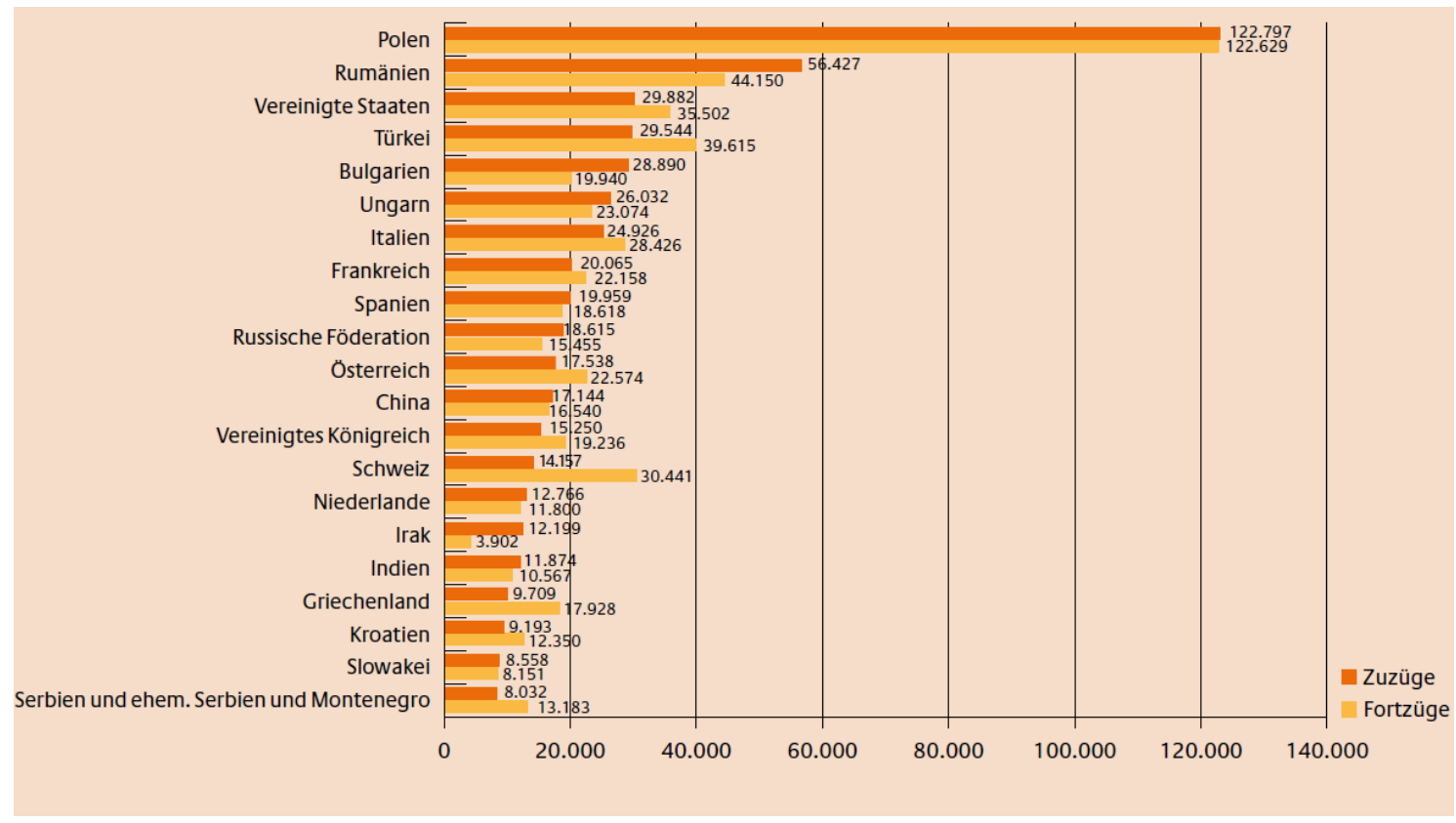

Quelle: Statistisches Bundesamt, entnommen aus Migrationsbericht, 2011, S. 24.

Bezogen auf das gesamte Migrationsgeschehen von rund 720.000 Zuzügen nach Deutschland und rund 730.000 Fortzügen aus Deutschland, entfielen mehr als 50\% der Zu- und Fortzüge auf das europäische Ausland. Polen stellte in diesem Zusammenhang etwa 1/3 aller europäischen Fälle dar. ${ }^{6}$

Für die gesetzliche Rentenversicherung sind allerdings nicht nur die Gesamtzahlen der Migration von Bedeutung, sondern in besonderem Maße die soziodemografischen Merkmale. Hier zeigt sich, dass etwa drei Viertel aller zuziehenden Personen unter 40 Jahre alt und damit ganz überwiegend im erwerbsfähigen Alter sind. ${ }^{7}$ Das bedeutet, dass das Migrationsgeschehen zu einer Verjüngung unserer Altersstruktur beiträgt.

5 Vgl. Migrationsbericht des Bundesamtes für Migration und Flüchtlinge im Auftrag der Bundesregierung, Januar 2011 für das Berichtsjahr 2009, insbesondere S. $24 \mathrm{f}$.

6 Vgl. Migrationsbericht (Fußn. 5), S. 21 ff.

7 Vgl. Migrationsbericht (Fußn. 5), S. 32. 
Das beschriebene Wanderungsgeschehen führt zu vielerlei praktischen Auswirkungen bei Arbeitgebern und Behörden, aber insbesondere auch bei den Sozialversicherungsträgern. So hat sich beispielsweise die Anzahl von Versicherungskonten bei der Deutschen Rentenversicherung (DRV) als Träger der gesetzlichen Rentenversicherung für polnische Wanderarbeitnehmer, d.h. für Personen, die zum Zwecke der Erwerbstätigkeit ins Ausland gehen bzw. gegangen sind, allein von Januar 2011 bis Juni 2011 um ca. 52.000 auf insgesamt rund 1.530 .000 erhöht. ${ }^{8}$ Etwa $75 \%$ dieser Konten werden derzeit auf Regionalebene und hier im Wesentlichen bei der DRV Berlin-Brandenburg als zuständiger Verbindungsstelle geführt. ${ }^{9}$ Da die neu hinzukommenden Konten nach der gesetzlich vorgeschriebenen Quote gem. § 127 Abs. 2 Satz 1 Nr. 1 SGB VI zu 55\% auf die Regionalträger, zu 40\% auf die DRV Bund und zu 5\% auf die DRV KBS verteilt werden, wird sich auf lange Sicht eine entsprechende Verschiebung der prozentualen Anteile ergeben. ${ }^{10}$

Abbildung 2: Entwicklung der durchschnittlichen monatlichen Rentenantragseingänge mit Bezug zu Polen bei der DRV Berlin-Brandenburg

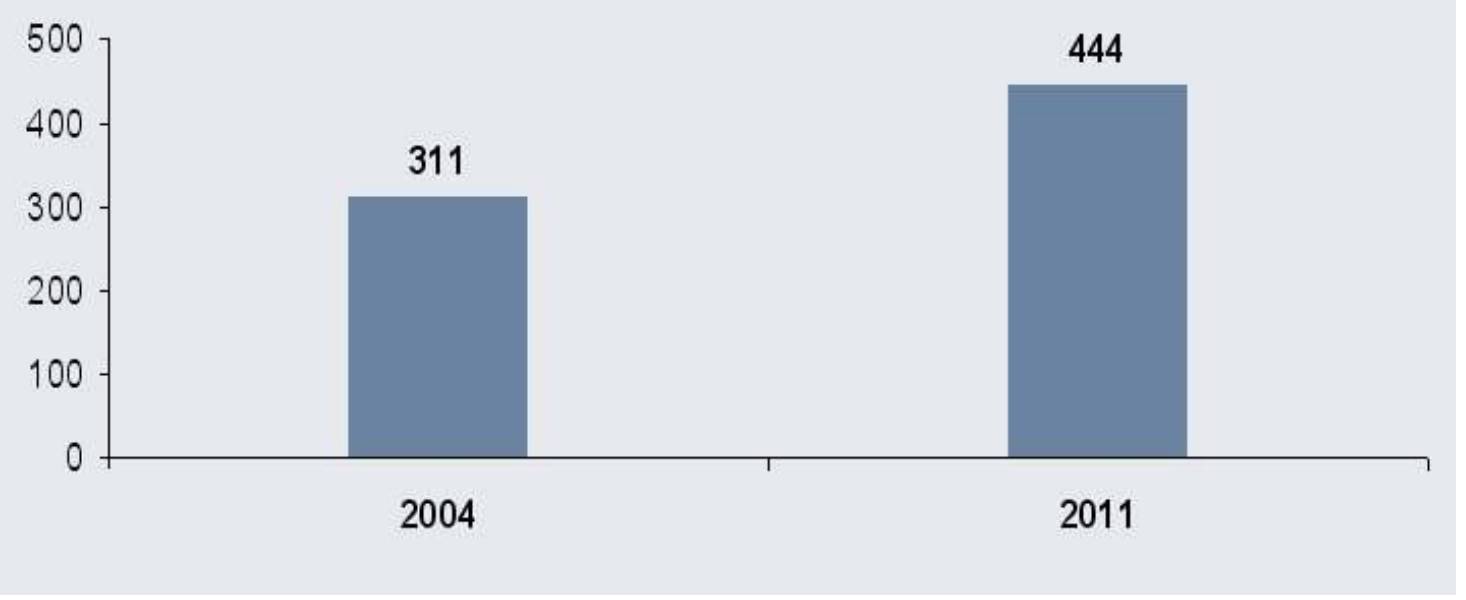

Quelle: DRV Berlin-Brandenburg.

Parallel zu dieser Entwicklung auf der Erwerbstätigenseite wächst außerdem die Zahl von Rentenleistungsanträgen von Versicherten, die neben deutschen auch polnische Zeiten haben bzw. im jeweils anderen Staat wohnen. So hat sich der diesbezügliche

8 Quelle: DRV Bund, 2011.

9 Obgleich die DRV Berlin-Brandenburg auf Regionalebene Verbindungsstelle zu Polen ist, werden die Einzelfälle nach dem deutsch-polnischen Rentenabkommen vom 09.10.1975 (DPSVA 1975) grundsätzlich nach dem Wohnortprinzip auch bei anderen Regionalträgern zuständigkeitshalber bearbeitet und die Versicherungskonten somit dort geführt.

$10 \mathrm{Zu}$ beachten ist hierbei, dass die im Konto dokumentierte Staatsangehörigkeit das Kriterium bei der Bestimmung von Versicherungskonten für Wanderarbeitnehmer ist und nicht das Zuzugsland. Allerdings zeigt sich gerade bei Saisonarbeitern eine hohe Übereinstimmung von beiden Merkmalen. 
Rentenantragseingang bei der DRV Berlin-Brandenburg seit dem EU-Beitritt Polens um mehr als 40\% auf deutlich über 5.000 im Jahr erhöht. Die Anzahl von Leistungsgewährungen nach Polen hat sich im gleichen Zeitraum sogar mehr als verdoppelt (2010: rund 4.900 Renten nach Polen). Bei der Nachfrage nach Leistungen zur Rehabilitation ist zwar ebenfalls eine Steigerung, allerdings (noch) in deutlich geringerem Ausmaß zu verzeichnen.

Aus der verstärkten Wanderungsbewegung zwischen Deutschland und Polen resultiert nicht nur eine Zunahme der Verwaltung von Versicherungskonten sowie der Bearbeitung entsprechender Leistungsanträge, sondern auch eine Zunahme des Beratungsbedarfs bei Versicherten, Arbeitgebern und Leistungsbeziehern sowie eine Zunahme von Anträgen auf Ausstellung von sog. Bescheinigungen A1 (vgl. Anlage). ${ }^{11}$ Die Bescheinigung A1 („Entsendebescheinigung“) ist eine Bescheinigung über die anzuwendenden Rechtsvorschriften in Anwendung des Unionsrechts. Sie hat zum 01.05.2010 die Bescheinigung E101 abgelöst, die grundsätzlich nur noch im Verhältnis zu den EWRStaaten und der Schweiz weiter gilt.

Infobox 1: Zuständigkeit bei Antragstellung der Bescheinigung A1

Antragsteller der Bescheinigung A1 sind Personen, die z.B. in Deutschland tätig sind und ihre Tätigkeit im Rahmen der Entsendung für eine bestimmte Zeit (maximal 24 Monate) im europäischen Ausland oder gewöhnlich in mehreren europäischen Staaten ausüben möchten. ${ }^{12}$ Für die Bearbeitung solcher Anträge ist in Deutschland vorrangig die Zuständigkeit der jeweiligen gesetzlichen Krankenversicherung gegeben; liegt allerdings kein gesetzlicher Krankenversicherungsschutz vor, so ergibt sich die Zuständigkeit der Deutschen Rentenversicherung, sofern nicht eine berufsständische Versorgungseinrichtung (z.B. für Ärzte) betroffen ist.

Insbesondere die Öffnung des deutschen Arbeitsmarktes zum 01.05.2011 hat zu einer sprunghaften Erhöhung der Antragszahlen von Entsendebescheinigungen bei der Deutschen Rentenversicherung geführt. Allein in der Zeit von Mai 2011 bis September 2011 sind bei der DRV Berlin-Brandenburg mit rund 300 Anträgen (ohne Sammelanträge) so viele Anträge eingegangen wie im gesamten Vorjahr.

\section{Anmerkungen zu den Rechtsanwendungen}

Die Bundesrepublik Deutschland und die Republik Polen haben bereits vor dem EUBeitritt Polens zwei Sozialversicherungsabkommen geschlossen, die für Rentenansprüche von Bedeutung waren bzw. zum Teil noch sind: das Abkommen vom 09.10.1975

11 Vgl. hierzu u. a. Bundesagentur für Arbeit, Erste Ergebnisse zu Auswirkungen der uneingeschränkten Arbeitnehmerfreizügigkeit seit 1. Mai, Pressemitteilung vom 27.07.2011, veröffentlicht in Die Beiträge 9/2011, S. 522 oder unter www.arbeitsagentur.de.

12 Vgl. Schüren, Die neue Entsendebescheinigung A-1 und die Voraussetzungen ihrer Erteilung, NZS 2011, S. 121. 
über Renten- und Unfallversicherung (DPSVA 1975; andernorts auch DPRA 1975 genannt) und das Abkommen vom 08.12.1990 über Soziale Sicherheit (DPSVA 1990). ${ }^{13}$

Mit dem Beitritt Polens zur Europäischen Union erhielt allerdings das Unionsrecht und somit seit 01.05.2004 die Verordnungen (EWG) Nr. 1408/71 bzw. 574/72 und seit deren Inkrafttreten am 01.05.2010 die neuen Verordnungen (EG) Nr. 883/2004 bzw. 987/2009 Geltung auch für die sozialversicherungsrechtlichen Beziehungen zwischen Deutschland und Polen. ${ }^{14}$ Jedoch lösen diese Verordnungen die bestehenden Abkommen nicht vollständig ab. Denn durch die Eintragung in den Anhangsregelungen zum Unionsrecht sind das DPSVA 1975 insgesamt sowie die dazu bestehende Durchführungsvereinbarung vom 11.01.1977 weiterhin anzuwenden. ${ }^{15}$ Die Eintragung in den Anhang beinhaltet dabei das DPSVA 1975 nach Maßgabe des Art. 27 Abs. 2 bis 4 DPSVA 1990, der als Übergangsbestimmung die Anwendung des DPSVA 1975 in Zuzugsfällen bis 31.12.1990 bzw. ausnahmsweise bis 30.06.1991 regelt. Durch die Eintragung dieser Bestimmung wird das bisherige Abgrenzungskriterium „Zuzugsdatum des Berechtigten“ für die Frage der Anwendung des DPSVA 1975 oder DPSVA 1990 auf die künftige Frage der Anwendung des DPSVA 1975 oder des europäischen Verordnungsrechts übertragen. Das DPSVA 1975 ist somit ggf. kumulativ mit all seinen Besonderheiten zu berücksichtigen. ${ }^{16}$

Im Verhältnis des DPSVA 1975 zum europäischen Verordnungsrecht ist dabei insbesondere Folgendes zu beachten:

- Rentenleistungen nach dem DPSVA 1975 werden nach dem sog. Eingliederungsprinzip gezahlt. ${ }^{17}$ Das Eingliederungsprinzip besagt, dass Berechtigte mit Wohnsitz in Polen ihre Rente auch aus deutschen Zeiten ausschließlich nach polnischen Rechtsvorschriften vom polnischen Versicherungsträger erhalten. Gegenüber der Deutschen Rentenversicherung bestehen insoweit keine Leistungsansprüche. Hingegen werden polnische Zeiten bei Wohnsitz in Deutschland wie deutsche Zeiten angerechnet. Der Berechtigte erhält damit aus allen Beiträgen vom Versicherungsträger seines Wohnsitzstaates eine Gesamtrente.

- Im Gegensatz dazu werden Leistungen nach dem Unionsrecht grundsätzlich nach dem Leistungsexportprinzip erbracht. Für die Berechnung der Rente gilt danach der Grundsatz, dass jeder Mitgliedstaat die Rente nur aus seinen eigenen Zeiten und nach seinen Rechtsvorschriften zahlt. Eine Zusammenrechnung von Versi-

13 Vgl. zu den Abkommen: www.deutsche-rentenversicherung-regional.de, Rechtliche Arbeitsanweisungen und Gesetzestexte.

14 Vgl. zu den Verordnungen (insbesondere zu den Rentenkapiteln): Hauschild, in: Hauck/Noftz (Hrsg.), EU-Sozialrecht Kommentar, 2010.

15 Vgl. Anhang II, Deutschland-Polen, Buchst. a Verordnung (EG) Nr. 883/2004 bzw. Anhang III, 19. Deutschland-Polen, Buchst. a Verordnung (EG) Nr. 1408/71.

16 Vgl. DRV Bund (Hrsg.), Das deutsch-polnische Abkommen vom 9. Oktober 1975, 2007, S. 4 ff., 18 ff. (Download unter www.deutsche-rentenversicherung-bund.de).

17 Vgl. Poletzky/Plaum, Sozialversicherungsabkommen zwischen Deutschland und Polen vom 09.10.1975, Nachtrag zur 2. Auflage der Polenbroschüre 1998, Teil C, Abschnitt 5.1. 
cherungszeiten erfolgt nur für die Erfüllung der Mindestversicherungszeiten und der besonderen versicherungsrechtlichen Voraussetzungen. Die Zahlung einer Gesamtrente durch einen Staat unter Berücksichtigung der Versicherungszeiten in den anderen Staaten erfolgt nicht.

- Das Unionsrecht beinhaltet im Unterschied zum DPSVA besondere Regeln zur Rentenberechnung, nach denen Versicherungszeiten der anderen Mitgliedstaaten auch Einfluss innerhalb der deutschen Rentenversicherung haben können (anteilige Rentenberechnung).

- Der sachliche Geltungsbereich des DPSVA 1975 erfasst auf polnischer Seite nach Maßgabe der dort geltenden Rechtsvorschriften nur Versicherungszeiten als Arbeitnehmer (einschließlich Bergleute und Eisenbahner), hingegen grundsätzlich nicht Zeiten als Nichtarbeitnehmer (Selbständige, Landwirte, Uniformträger). Zeiten als Nichtarbeitnehmer können daher prinzipiell nur dann abkommensrelevant sein, wenn der Versicherte zuletzt in Polen Arbeitnehmer gewesen ist. Vom Geltungsbereich des Unionsrechts werden dagegen Versicherungszeiten als Arbeitnehmer einschließlich Beamte sowie als Selbständige einschließlich Landwirte erfasst. Aufgrund des weiter gefassten Geltungsbereichs kommt danach auch eine Berücksichtigung zurückgelegter polnischer Versicherungszeiten z.B. als Selbständiger, Landwirt, Uniformträger, Richter, Staatsanwalt etc. in Betracht.

Damit gelten bei Feststellung der Rente in Deutschland für in Polen zurückgelegte Zeiten weiterhin die Regelungen des DPSVA 1975 einschließlich des Zustimmungsgesetzes (ZustG) (sog. „FRG-Filter“) und im Übrigen die sonstigen Regelungen des Verordnungsrechts, sofern der Berechtigte unter die Anwendung des DPSVA 1975 fällt und auch sämtliche zurückgelegten Versicherungszeiten vom Abkommen erfasst werden. ${ }^{18}$

Sind darüber hinaus in Polen zurückgelegte Zeiten vorhanden, die künftig allein in Anwendung des Unionsrechts rechtserheblich sind, wird aus diesen nicht vom DPSVA 1975 erfassten polnischen Zeiten außerdem der polnische Versicherungsträger leistungspflichtig (sog. Mischfälle). In diesen Fällen wird eine anteilige Rentenberechnung nach dem Unionsrecht ausgelöst (kumulative Anwendung des DPSVA 1975 mit dem europäischen Verordnungsrecht). Mischfälle dieser Art können auch dann auftreten, wenn der grundsätzlich bis zum 31.12.1990 zugezogene Versicherte außer den Zeiten in Polen und Deutschland noch weitere Zeiten in einem anderen EU/EWR-Staat oder in der Schweiz zurückgelegt hat.

Für die Frage der Rechtsanwendung ergibt sich eine Besonderheit bei Ehepaaren. Denn in zahlreichen Fällen sind Ehepaare bis zum 31.12.1990 nicht gemeinsam von Polen nach Deutschland verzogen. Im Hinterbliebenenrentenfall liegt dann auch für den

18 Das Zustimmungsgesetz (ZustG) wurde am 12. März 1976 verabschiedet (BGBl. II S. 393) und zuletzt durch Artikel 20 des Rentenreformgesetzes 1992 vom 18.12.1989 (BGBl. I S. 2261, 2375; 1990 S. 1337) geändert (vgl. www.deutsche-rentenversicherung-regional.de, Rechtliche Arbeitsanweisungen und Gesetzestexte). 
rechtzeitig bis zum 31.12.1990 (ausnahmsweise bis 30.06.1991) zugezogenen Berechtigten ein sog. Mischfall vor, wenn von dem verstorbenen Versicherten zusätzlich noch weitere Versicherungszeiten ab 01.01.1991 in Polen zurückgelegt worden sind. ${ }^{19}$

Werden hingegen sämtliche polnische Zeiten nicht vom DPSVA 1975 erfasst und findet das DPSVA 1975 keine Anwendung, gilt das europäische Verordnungsrecht ohne Besonderheiten.

Für die Rechtsanwendung ergeben sich damit folgende Fallgruppen:

- reine Anwendung von europäischem Verordnungsrecht,

- kumulative Anwendung DPSVA 1975 und europäisches Verordnungsrecht und

- reine DPSVA 1975-Anwendung.

In der Praxis sind die Fallgruppen von unterschiedlicher Bedeutung. Während auf eine reine Anwendung von europäischem Verordnungsrecht auf Regionalebene der Deutschen Rentenversicherung bundesweit rund 60\% entfallen (mit steigender Tendenz), beläuft sich der Anteil auf reine DPSVA 1975-Anwendung auf rund 30\% mit fallender Tendenz. Der Anteil von Mischfällen ist bei rund 10\% stabil.

Infobox 2: Anmerkungen zur Bedeutung des DPSVA 1990

Im Gegensatz zum DPSVA 1975 ist das DPSVA 1990 nach Art. 8 der Verordnung (EG) Nr. 883/2004 bzw. Art. 6 der Verordnung (EWG) Nr. 1408/71 nachrangig zum Unionsrecht und findet daher seit 01.05.2004 in der Praxis nur noch in bestimmten Ausnahmefällen z.B. bei Drittstaatsangehörigen (mit deutschen und polnischen Versicherungszeiten) mit gewöhnlichem Aufenthalt außerhalb der EU-Staaten und hinsichtlich Altfällen (d.h. Fällen, die seinerzeit nach dem DPSVA 1990 festgestellt und die nicht nach Unionsrecht neu festgestellt wurden) Anwendung.

Vor dem Hintergrund der Rechtsgrundlagen stellen sich in der Praxis eine Reihe von Fragen zur Anwendung. Für Fälle in Zeiten der Erwerbstätigkeit steht dabei die Frage nach der Anwendung der zutreffenden Rechtsvorschriften (Stichwort „Kollisionsnormen“) im Mittelpunkt, während für die Zeiten des Leistungsbezugs Fragen zu den Auswirkungen bei Verzug eines Berechtigten von wesentlicher Bedeutung sind.

\section{Zentrale Fragestellungen aus Sicht der Praxis}

\section{Fragen im Rahmen der Erwerbsphase}

Für die Praxis der Rentenversicherung sind im Rahmen der Erwerbsphase die Kollisionsnormen von besonderer Bedeutung. Als Kollisionsnormen werden die Regelungen im Titel II, d.h. Art. 11 ff. der Verordnung (EG) Nr. 883/2004 bezeichnet. Sie bestim-

19 Eine Ausnahme sieht hierbei vor, dass diese Zeiten - längstens bis zum 30.06.1991 - wie die Zeiten bis 31.12.1990 ggf. nach dem DPSVA 1975 beurteilt werden, wenn der verstorbene Versicherte die Voraussetzungen des Art. 27 Abs. 4 DPSVA 1990 erfüllt hat. 
men, welche Rechtsvorschriften bei grenzüberschreitender Beschäftigung oder selbständiger Erwerbstätigkeit anzuwenden sind. Sinn und Zweck der Kollisionsnormen ist es, Versicherungslücken und Doppelversicherungen zu vermeiden.

Grundsätzlich unterliegen Personen, für die die o.g. Verordnung (EG) gilt, nur den Rechtsvorschriften eines Mitgliedstaates, und zwar bei Beschäftigung oder selbständiger Erwerbstätigkeit denjenigen Rechtsvorschriften des Mitgliedsstaates, in dem die Beschäftigung oder selbständige Erwerbstätigkeit ausgeübt wird. ${ }^{20}$ Damit findet grundsätzlich das Territorialitätsprinzip (Art. 11 Abs. 3 Buchst a Verordnung (EG) 883/2004) Anwendung. Allerdings gibt es eine Reihe von Ausnahmen.

Eine wesentliche Ausnahme ist die Entsendung nach Art. 12 Verordnung (EG) Nr. 883/2004. ${ }^{21}$ Mit ihr soll die Arbeitnehmerfreizügigkeit gewährleistet und zugleich unnötige Bürokratie vermieden werden. Eine Entsendung von Beschäftigten nach Art. 12 Abs. 1 Verordnung (EG) 883/2004 liegt demnach vor, wenn

- der Beschäftigte von einem Unternehmen mit Sitz z. B. in Deutschland aus Deutschland vorübergehend in einen anderen Mitgliedstaat entsandt wird,

- die Entsendung zeitlich auf maximal 24 Monate befristet ist und

- die Entsendung im Rahmen des inländischen (deutschen) Beschäftigungsverhältnisses weiter ausgeübt wird.

Eine entsprechende Regelung gibt es nach Art. 12 Abs. 2 Verordnung (EG) 883/2004 auch für Personen, die ihre selbständige Erwerbstätigkeit vorübergehend in einen anderen Mitgliedstaat verlagern. In diesen Fällen liegt eine Entsendung vor, wenn

- im anderen Mitgliedstaat eine ähnliche Tätigkeit ausgeübt wird,

- vor der Entsendung im Entsendestaat eine nennenswerte Tätigkeit erbracht wurde,

- die Infrastruktur im Entsendestaat aufrecht erhalten wird,

- vor der Entsendung die deutschen Rechtsvorschriften über soziale Sicherheit galten und

- die Tätigkeit im Voraus auf maximal 24 Monate befristet ist.

Auch in Fällen, in denen Personen eine Beschäftigung oder selbständige Erwerbstätigkeit in zwei oder mehreren Mitgliedstaaten ausüben, sind grundsätzlich die Rechtsvorschriften nur eines Mitgliedstaats anzuwenden. Die Personen werden dabei sozialversicherungsrechtlich so behandelt, als wenn sie ihre gesamte Erwerbstätigkeit in diesem Mitgliedstaat absolvieren. Entsprechende Regelungen hierzu enthält Art. 13 Verordnung (EG) 883/2004.

Danach ist bei Personen, die in mehreren Mitgliedstaaten eine Beschäftigung oder selbständige Tätigkeit ausüben, das Recht des Wohnstaates anzuwenden, wenn dort ein

20 Vgl. Art. 11 Abs. 1 und Abs. 3 Buchst. a Verordnung (EG) Nr. 883/2004.

21 Zur Entsendungsthematik siehe u. a.: Die Rechtsvorschriften, die für Erwerbstätige in der Europäischen Union (EU), im Europäischen Wirtschaftsraum (EWR) und der Schweiz gelten, Praktischer Leitfaden der Europäischen Kommission, 01/2011. 
wesentlicher Teil der Beschäftigung/Tätigkeit erbracht wird (Art. 13 Abs. 1 Buchst. a bzw. Abs. 2 Buchst. a Verordnung (EG) 883/2004). Wird der wesentliche Teil der Beschäftigung/Tätigkeit nicht im Wohnstaat erbracht, sind für die Anwendung der Rechtsvorschriften bei Beschäftigung der Sitz des Unternehmens/Arbeitgebers und bei selbständiger Erwerbstätigkeit der Ort des Mittelpunkts der selbständigen Tätigkeiten für die Rechtsanwendung ausschlaggebend (Art. 13 Abs. 1 Buchst. b bzw. Abs. 2 Buchst. b Verordnung (EG) 883/2004).

Bei Personen, die in verschiedenen Mitgliedstaaten sowohl eine Beschäftigung als auch eine selbständige Tätigkeit ausüben, ist das Recht des Mitgliedstaats anzuwenden, in dem die Beschäftigung ausgeübt wird (Art. 13 Abs. 3 Verordnung (EG) 883/2004). Werden Beschäftigungen in mehreren Mitgliedstaaten ausgeübt, findet wiederum Art. 13 Abs. 1 Buchst. b Verordnung (EG) 883/2004 Anwendung.

Bei der Anwendung von deutschem Sozialversicherungsrecht sind hierbei die besonderen Regelungen in Bezug auf das Vorliegen von Versicherungspflicht bzw. Versicherungsfreiheit zu beachten: So liegt z. B. bei Ausübung einer selbständigen Tätigkeit in Deutschland grundsätzlich keine Versicherungspflicht in der gesetzlichen Rentenversicherung vor. Kraft Gesetzes sind nur selbständig Tätige in der gesetzlichen Rentenversicherung versicherungspflichtig ( 2 SGB VI), die ein besonderes Schutzbedürfnis haben, so z. B. Lehrer und Erzieher, Hebammen und Entbindungspfleger, Künstler und Publizisten, Hausgewerbetreibende, Handwerker und Selbständige mit einem Auftraggeber. 22

Eine weitere Besonderheit in Deutschland ist die geringfügige Beschäftigung (sog. Minijob). ${ }^{23}$ Eine Beschäftigung kann aufgrund der Höhe des Arbeitsentgelts von maximal 400 EUR monatlich (geringfügig entlohnte Beschäftigung; § 8 Abs. 1 Nr. 1 SGB IV) oder wegen der kurzen Dauer von nicht mehr als zwei Monaten bzw. 50 Arbeitstagen im Kalenderjahr (kurzfristige Beschäftigung; § 8 Abs. 1 Nr. 2 SGB IV) geringfügig sein. Der Arbeitnehmer zahlt in diesen Fällen keine Sozialversicherungsbeiträge; die Pauschalabgaben zur Sozialversicherung werden vom Arbeitgeber übernommen. Bei dieser geringfügig entlohnten Beschäftigung betragen die Pauschalabgaben zur Rentenversicherung 15\% und zur Krankenversicherung 13\% sowie die einheitliche Pauschalsteuer 2\%. Durch diese Regelungen begründen geringfügige Beschäftigungen zunächst keinen Versicherungsschutz. Allerdings können geringfügig Beschäftigte den Beitrag zur gesetzlichen Rentenversicherung auf den regulären Beitragssatz von derzeit 19,6\% aufstocken (sog. „Aufstocker“) und erhalten dadurch neben dem vollständigen Versi-

22 Vgl. hierzu u. a. Dünn/Fasshauer, in: Handbuch zur Altersvorsorge, 2009, S. 225 ff.

$23 \mathrm{Vgl}$. zu diesen sog. „Geringfügigen Beschäftigungsverhältnissen“ ausführlich die Informationen im Internet unter: www.minijobzentrale.de. 
cherungsschutz u. a. auch die Möglichkeit, die staatlich geförderte Altersvorsorge („Riester-Rente“) in Anspruch nehmen zu können. ${ }^{24}$

Die Anwendung von deutschem Sozialversicherungsrecht im Bereich der geringfügigen Beschäftigung, wie auch bei Selbständigkeit scheint aufgrund der geringeren Beitragsleistungen bzw. des vollständigen Wegfalls der Versicherungspflicht für Einzelne vorteilhaft (vgl. Beispiel 1 und Beispiel 2). Schließlich vergünstigen sich für die Betroffenen zumindest vermeintlich die Arbeitskosten und sie können ihre Arbeitsleistung zu deutlich geringeren Kosten anbieten. Gerade im Hinblick auf das deutsch-polnische Migrationsgeschehen zeigt sich diesbezüglich eine zunehmende, sozialpolitisch problematische Dynamik, da von den Betroffenen vielfach nicht berücksichtigt wird, dass sich der nicht bestehende Versicherungsschutz bzw. die nicht vorliegende Versicherungspflicht im Leistungsfall nachteilig für sie auswirken. Das betrifft die Leistungshöhe im Alter ebenso, wie beispielsweise den Anspruch auf Leistungen zur Teilhabe oder aber auch den aktuellen Krankenversicherungsschutz. Damit ergibt sich nicht nur eine Schwächung des Sozialversicherungssystems als Ganzes aufgrund der fehlenden Einnahmen, sondern vor allem auch eine Verschlechterung der sozialen Absicherung des Einzelnen.

Beispiel 1: Umgehung der Sozialversicherungspflicht im Wohnstaat durch geringfügige Beschäftigung in Deutschland

Ausgangslage:

Die betreffenden Personen wohnen in Polen und üben dort eine selbständige Tätigkeit aus. Gleichzeitig wird eine geringfügig entlohnte Beschäftigung (Minijob) für ein in Deutschland ansässiges Unternehmen mit Arbeitsort beispielsweise sowohl Tschechien als auch in der Slowakei ausgeübt. Das in Deutschland ansässige Unternehmen beschäftigt dabei fast ausschließlich Arbeitnehmer, die nicht in Deutschland tätig sind. Somit ist eine Geschäftstätigkeit des Unternehmens in Deutschland zweifelhaft.

Rechtslage:

Nach derzeitiger Rechtsauffassung gilt gem. Art. 13 Abs. 1 Buchst. b Verordnung (EG) Nr. 883/2004 deutsches Recht sowohl für den Minijob als auch für die selbständige Tätigkeit in Polen. ${ }^{25}$ Sozialversicherungsbeiträge werden nicht bzw. nur vermindert geleistet.

24 Vgl. hierzu mit zahlreichen weiteren Literaturhinweisen Fasshauer/Rieckhoff, Kapitalanlagen, die nach dem Altersvermögensgesetz gefördert werden (sog. ,Riester-Rente“), in: Handbuch zur Altersvorsorge, 2009, S. $721 \mathrm{ff}$.

25 Diese Rechtsauffassung ist allerdings noch nicht abschließend geklärt. Es fand in einem derartigen Fall zwischen der DRV Bund und dem polnischen Träger der allgemeinen Rentenversicherung Zakład Ubezpieczen Społecznych (ZUS) ein Verfahren nach Art. 16 Abs. 4 Verordnung (EG) Nr. 987/2009 zur endgültigen Festlegung der Rechtsvorschriften statt. Eine abschließende Klärung wurde bisher nicht herbeigeführt. 
Beispiel 2: Umgehung der Sozialversicherungspflicht im Wohnstaat durch selbständige Tätigkeit in Deutschland

Ausgangslage:

Wohnstaat der selbständig tätigen Person ist Polen, aber die selbständige Tätigkeit (Gewerbeanmeldung) erfolgt gewöhnlich in Deutschland. Der Selbständige verlagert dabei seine selbständige Tätigkeit vorübergehend (maximal 24 Monate) in einen anderen Mitgliedstaat. In Deutschland besteht für die selbständige Tätigkeit keine Versicherungspflicht in der gesetzlichen Rentenversicherung.

Rechtslage:

Für die Dauer der Verlagerung/Entsendung der selbständigen Tätigkeit im anderen Mitgliedstaat ist weiterhin deutsches Recht anzuwenden. Somit besteht im Ergebnis weiterhin Versicherungsfreiheit in der Sozialversicherung nach deutschem Recht.

Beide Beispiele zeigen, dass es rechtliche Lücken gibt, die durch geschickte Fallkonstellationen ausgenutzt werden können - zu Lasten der Sozialversicherung und zu Lasten des Einzelnen. Mögliche Optionen zur Schließung dieser Lücken reichen von Einzelregelungen bis zur Einführung einer sog. Erwerbstätigenversicherung in Deutschland, d.h. der Ausweitung der gesetzlichen Rentenversicherungspflicht auf Beamte, Selbständige und Minijobber. ${ }^{26}$

\section{Fragen im Rahmen des Rentenbezugs}

\section{Vorbemerkungen}

Ein Wegzug aus Deutschland ins Ausland kann für Rentenbezieher mit erheblichen Einbußen verbunden sein. Dies, obwohl mittlerweile europaweit die Angleichung der Rechtssysteme und die Beseitigung von Hemmnissen deutlich vorangeschritten ist. Die Einbußen aufgrund eines Umzugs ergeben sich dabei aus speziellen rechtlichen Besonderheiten und/oder aufgrund historisch gewachsenem Sonderrecht.

Für die Auswirkungen eines Wohnsitzwechsels bei Rentenbezug ist entscheidend, mit welchen Zeiten die Person und wohin die Person verzieht. In Bezug auf Polen sind die unter Kapitel 3 beschriebenen verschiedenen Rechtsanwendungen und hierbei insbesondere die Fälle nach dem DPSVA 1975 und Fälle von Personen, auf die das Fremdrentengesetz (FRG) Anwendung findet, also Personen mit sog. FRG-Berechtigung, von Interesse.

26 Vgl. hierzu u. a. Grünbuch „Angemessene, nachhaltige und sichere europäische Pensions- und Rentensysteme“", KOM (2010) 365, Juli 2010, Ziffer 2.2, Absatz 5. 
2. Berechtigung auf Leistungen nach dem deutsch-polnischen Sozialversicherungsabkommen von 1975 (DPSVA 1975)

Voraussetzung für die Anwendung des DPSVA 1975 ist wie oben beschrieben, dass die berechtigte Person ihren gewöhnlichen Aufenthalt in Deutschland oder Polen grundsätzlich vor dem 01.01.1991 begründet hat und dieser seither unverändert fortbesteht. Ein Verzug in den jeweiligen anderen Staat oder in einen Drittstaat führt zum unwiderruflichen Wegfall der Abkommensanwendung. ${ }^{27}$ Der Rentenanspruch richtet sich danach rein nach dem europäischen Verordnungsrecht.

Dies bedeutet, dass nach Wegfall der Anwendung des DPSVA 1975 mit seinem Eingliederungsprinzip durch Verzug in einen anderen Staat, ein deutscher Rentenanspruch nur aus deutschen Zeiten einschließlich ggf. FRG-Zeiten gewährt werden kann. Dies kann zum Wegfall oder zur Minderung des deutschen Rentenanspruchs führen (vgl. Beispiele 3 und 4). Der Wegfall ergibt sich, wenn neben den vom DPSVA 1975 erfassten Zeiten keine deutschen Zeiten einschließlich nach dem FRG vorhanden sind. Eine Minderung ergibt sich, wenn deutsche Zeiten einschließlich nach dem FRG vorhanden sind. Gleichzeitig besteht aus den polnischen Zeiten ab Verzug ein Anspruch gegenüber dem polnischen Träger in Anwendung des europäischen Verordnungsrechts.

Beispiel 3: Auswirkungen eines Verzugs von Deutschland nach Polen in der Rentenbezugsphase ohne FRG-Berechtigung

Ausgangslage:

- der Berechtigte wohnt seit 1988 ununterbrochen in Deutschland

- das DPSVA 1975 ist anwendbar

- es liegt eine rein polnische Versicherungsbiografie vor

- alle zurückgelegten Zeiten werden vom DPSVA 1975 erfasst

- es liegt keine FRG Berechtigung vor

- die deutsche Rente beträgt in Anwendung des DPSVA 1975 = 1.000,- EUR mtl.

- Verzug nach Polen erfolgt im Mai 2011

Auswirkungen:

- die deutsche Rente in Höhe von 1.000,- EUR nach dem DPSVA 1975 fällt zum 31.05.2011 unwiderruflich weg

- ab 01.06.2011 besteht kein deutscher Rentenanspruch mehr, da keine deutschen Zeiten vorhanden sind

- ein polnischer Rentenanspruch ist vom polnischen Träger zu prüfen. Ggf. erhält der Berechtigte zumindest die polnische Mindestaltersrente von z. Zt. $728,18 \mathrm{Zt} \mathrm{mtl.}{ }^{28}$, dies entspricht etwa $167,-\mathrm{EUR}^{29} \mathrm{mtl}$.

27 Vgl. Das deutsch-polnische Abkommen vom 9. Oktober 1975 (Fußn. 16), S. 4.

28 Vgl. Sozialversicherung in Polen, Informationen, Fakten, Hrsg. ZUS, Warschau, 2011, S. 39 (Download unter www.zus.pl).

29 EURO-Referenzkurs, Deutsche Bundesbank, Stand 07.11.2011. 


\section{Berechtigung auf Leistungen nach dem Fremdrentengesetz (FRG)}

Für Berechtigte auf Leistungen nach dem Fremdrentengesetz (FRG) sind die Folgen eines Verzuges seit dem EuGH-Urteil vom 18.12.2007 von geringerem Ausmaß. ${ }^{30}$ Bis zu diesem Zeitpunkt war der Export von Leistungen aus FRG-Zeiten nach den Auslandsrentenvorschriften der $\S \S 110 \mathrm{ff}$. SGB VI durch Eintragung in den Anhangsregelungen zum europäischen Verordnungsrecht (ebenfalls) ausgeschlossen. Seit der Europäische Gerichtshof $(\mathrm{EuGH})$ jedoch die Unvereinbarkeit dieser Vorschriften mit dem Freizügigkeitsprinzip und dem Leistungsexportgebot festgestellt hat und die Aufhebung der Wohnortklausel nach Art. 10 Verordnung (EG) Nr. 1408/71 bzw. Art. 8 Verordnung (EG) Nr. 883/2004 gilt ${ }^{31}$, sind die Renten bei Verzug in einen anderen EU/EWR-Staat sowie die Schweiz aus allen FRG-Zeiten und Reichsgebietszeiten zu ermitteln. ${ }^{32}$ Damit erfolgt ein Leistungsexport grundsätzlich in Höhe der Inlandsrente. Allerdings ist zu beachten, dass eine Absenkung nach § 22 Abs. 4 FRG auf 60\% vorgenommen wird und bei einer gleichzeitigen Rentengewährung aus dem Ausland ein Ruhen der deutschen Rente nach § $31 \mathrm{FRG}$ zu prüfen ist.

Im Ergebnis resultiert daraus in den Fällen, in denen bis zum Verzug das DPSVA 1975 Anwendung fand, eine Minderung des deutschen Rentenanspruches (vgl. Beispiel 4). $\mathrm{Zu}$ einem vollständigen Wegfall wie in Beispiel 3 dargestellt kommt es nicht.

Beispiel 4: Auswirkungen eines Verzugs von Deutschland nach Polen in der Rentenbezugsphase mit FRG-Berechtigung

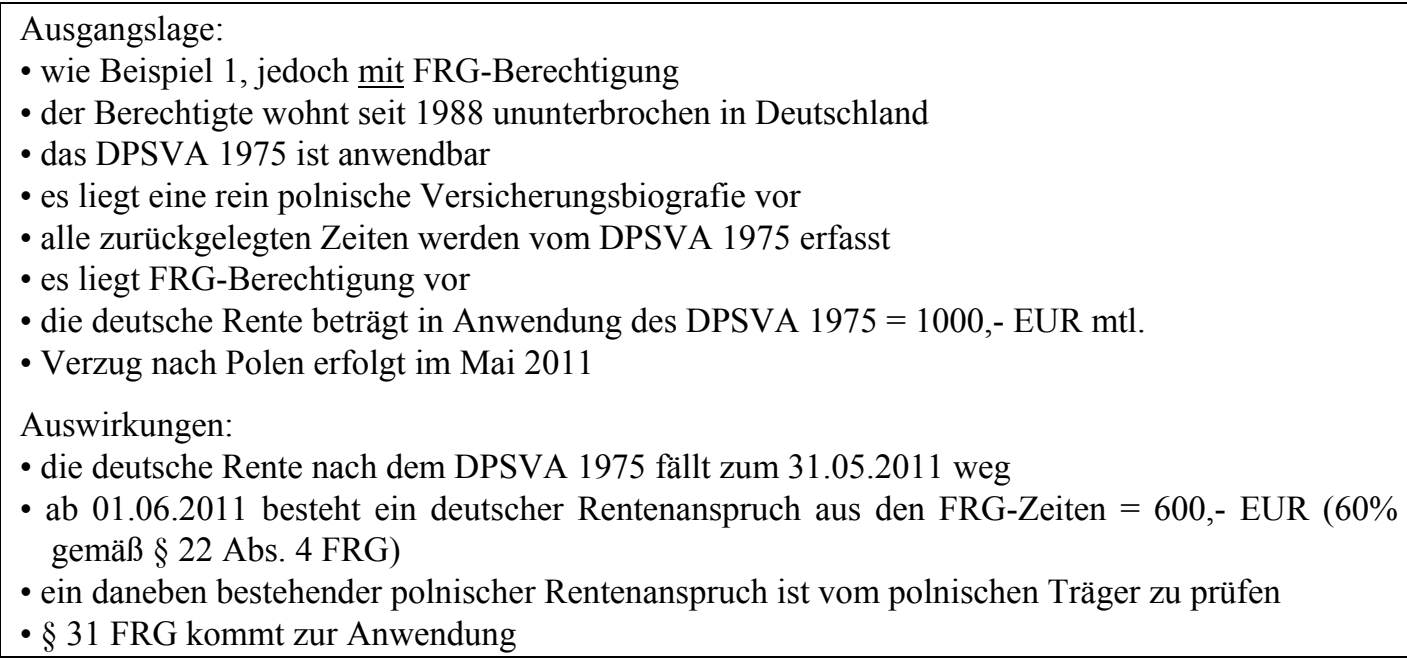

30 Vgl. EuGH-Urteil vom 18.12.2007, verbundene Rs. C-396/05 (Habelt), C-419/05 (Möser) und C450/05 (Wachter), Slg. 2007, I-11895.

31 Vgl. EuGH-Urteil vom 18.12.2007 (Fußn. 30), insbes. Rn. 81, 82, 85, 114.

32 Dies gilt auch bei gewöhnlichem Aufenthalt in einem anderen EU/EWR-Staat sowie der Schweiz, d.h. ohne Verzug aus Deutschland, Vgl. EuGH-Urteil vom 18.12.2007 (Fußn. 30), insbes. Rn. 97, 121. 
Neben den teilweise gravierenden Folgen eines Verzugs für den einzelnen Leistungsbezieher, ergeben sich bei einem Verzug von Deutschland nach Polen auch für den deutschen Träger der Rentenversicherung Verfahrensfragen. Diese basieren weitgehend auf der Problematik der Überzahlung, da in der Praxis trotz entsprechender Hinweise im deutschen Rentenbescheid der Verzug ins Ausland von den Berechtigten oftmals nicht oder aber erst verzögert mitgeteilt wird. Die Folgen sind erhöhter Verwaltungsaufwand wegen weiterer Ermittlungen und ggf. der Beitreibung von Forderungen (vgl. Kapitel $4.3)$.

\section{Weitere Entwicklung}

Die Folgen der in den Beispielen 3 und 4 veranschaulichten Rentenkürzungen werden vielfach als Hemmnis in der Inanspruchnahme von Freizügigkeit wahrgenommen. Sie berühren damit einen wesentlichen Kern des „europäischen Gedankens“. Die Auswirkungen werden deshalb weiter in der rechtlichen und auch politischen Diskussion bleiben. Die Europäische Union und insbesondere das koordinierende Unionsrecht sind kein starres Konstrukt, sondern stetig dem Wandel und der Anpassung unterworfen, so dass auch in dieser Hinsicht durchaus weitere Veränderungen nicht auszuschließen sind, wobei als Maßstab das im Art. 45 GRC verankerte Grundrecht auf Freizügigkeit gilt.

In diesem Zusammenhang ist das gegen Deutschland anhängige Vertragsverletzungsverfahren Nr. 2008/4941 der Europäischen Kommission aufmerksam zu verfolgen. ${ }^{33}$ In diesem Verfahren wird gerügt, dass in Anwendung des Art. 28 Abs. 1 Buchst. b des deutsch-bulgarischen SV-Abkommens vom 17.12.199734, der dem Bestandsschutz von Leistungsfällen nach dem Abkommen der ehemaligen DDR mit der ehemaligen Volksrepublik Bulgarien dient, sich der deutsche Rentenanspruch bei Verzug eines Rentners nach Bulgarien verringert. ${ }^{35}$ Diese Verringerung laufe dem Grundrecht auf Freizügigkeit und dem Leistungsexportgebot zuwider und sei zudem den dem EuGH-Urteil vom 18.12.200736 zugrunde liegenden Sachverhalten vergleichbar.

Nach Auffassung der Deutschen Rentenversicherung und der Bundesregierung unterscheiden sich die Abkommenssachverhalte jedoch wesentlich von den dem o.g. EuGH-Urteil zugrunde liegenden Sachverhalten. ${ }^{37}$ Nach dem o.g. deutsch-bulgarischen Abkommen ermittelten die Versicherungsträger beider Länder zunächst den theoretischen Betrag aus allen Versicherungszeiten in beiden Vertragsstaaten (sog. „Zunächst-

33 Vgl. Aufforderungsschreiben der Europäischen Kommission vom 22.03.2010, SG-Greffe (2010) D/3735 sowie Europäische Kommission, IP 11/420 vom 06.04.2011.

34 Vgl. Anhang III Teil A Nr. 2 Buchst. a Verordnung (EG) Nr. 1408/71 bzw. Anhang II Verordnung (EG) Nr. 883/2004.

35 Vgl. SV-Abkommen der DDR und Bulgarien vom 20.02.1953 in der Fassung der Zusatzvereinbarung vom 07.02.1973.

36 Vgl. EuGH-Urteil vom 18.12.2007 (Fußn. 30).

37 Vgl. Mitteilung der Regierung der Bundesrepublik Deutschland an die Europäische Kommission vom 7. Juni 2010. 
Rente"). Danach wurde der jeweilige Teilrentenbetrag in beiden Abkommensstaaten ermittelt. War die Summe der beiden Teilrenten jedoch geringer als die „ZunächstRente", so musste der Wohnsitzstaat zusätzlich den Differenzbetrag an den Berechtigten erbringen. Der Differenzbetrag führt somit bei Wohnsitz des Berechtigten in Deutschland zu einer „Gesamtrente“ auf dem „deutschen Niveau“, ohne dass die ausländischen Versicherungszeiten dabei - im Gegensatz zum FRG - in das deutsche Rentenversicherungssystem eingegliedert werden.

Allerdings ist derzeit nicht absehbar, ob sich die Europäische Kommission dieser Sichtweise anschließt. Für die Fragestellung des Leistungsbezugs bei Verzug von Deutschland nach Polen sind etwaige Auswirkungen des Vertragsverletzungsverfahrens daher gegenwärtig nicht einschätzbar.

\section{Beitreibung von Forderungen}

Der Wohnsitzwechsel ins Ausland führt vielfach zu Leistungsüberzahlungen. Allerdings kann es auch aus anderen Gründen zu Leistungsüberzahlungen kommen oder es können auch Beitragsforderungen des Rentenversicherungsträgers gegen Versicherte im Ausland bestehen. Hier normiert das neue Verordnungsrecht seit dem 01.05.2010 erstmals die Möglichkeit der Vollstreckung von Forderungen in anderen Mitgliedstaaten im Wege der Beitreibung gem. Art. 84 Verordnung (EG) Nr. 884/2004. ${ }^{38}$ Als wesentliches Element beinhaltet diese Vorschrift die gegenseitige Anerkennung von Vollstreckungstiteln, entweder unmittelbar oder durch Vollstreckbarkeitserklärung sowie die Gleichstellung der Forderungen. Die Einzelheiten zur Durchführung des Art. 84 Verordnung (EG) Nr. 884/2004 sind in den Art. 75 bis 85 Verordnung (EG) Nr. 987/2009 geregelt. Bestehende Rückforderungsmöglichkeiten im Wege des Ausgleichs nach Art. 71 Verordnung (EG) Nr. 987/2009 sind dabei vorrangig.

Die Vorschriften zur Beitreibung finden allerdings immer dann keine Anwendung, wenn die Forderung bereits verjährt ist (Art. 83 Abs. 1 Verordnung (EG) Nr. 987/2009), älter als fünf Jahre ist (Art. 82 Verordnung (EG) Nr. 987/2009) bzw. die Forderung nicht mehr als 350,- EUR beträgt. ${ }^{39}$ Diese Einschränkungen sind aus Überlegungen der Praktikabilität getroffen worden.

38 Vgl. u.a. Bourauel/Nagel/Petersen, in: DRV Bund (Hrsg.), Soziale Sicherheit in Europa - Rentenversicherung, 2011, S. 42 ff.

39 Nach Art. 75 Abs. 3 Verordnung (EG) Nr. 987/2009 wird eine Mindestschwelle für Beträge, für die ein Beitreibungsersuchen gestellt werden kann, von der Verwaltungskommission festgelegt. Obgleich noch keine endgültige Festlegung getroffen wurde, wird von den deutschen Trägern derzeit von einer Mindestschwelle i. H. v. 350 Euro ausgegangen; vgl. Gemeinsame Arbeitsanweisungen, Art. 75 Verordnung (EG) 987/2009, Abschnitt R4.1.2 (abrufbar unter www.deutsche-rentenversicherung-regional.de). 
Infobox 3: Wesentliche Inhalte der Art. 75 ff. Verordnung (EG) Nr. 987/2009 für die Beitreibung von Forderungen

- Zustellungsersuchen (Art. 77)

- Beitreibungsersuchen mit Titel (Art. 78)

- gegenseitige Anerkennung von Vollstreckungstiteln (Art. 79)

- Grenzen der Unterstützung z.B. darf die Forderung nicht älter als 5 Jahre sein (Art. 82)

- Sicherungsmaßnahmen (Art. 84)

- Grundsatz der unentgeltlichen Amtshilfe mit der Möglichkeit der Vereinbarung von Erstattungsmodalitäten im Einzelfall (Art. 85)

Im Ergebnis soll der ersuchte Träger die Forderung aus einem anderen Mitgliedstaat wie eine eigene Forderung behandeln. Die sich jeweils aus dem nationalen Recht ergebenden Grenzen der Beitreibung sind dabei zu beachten. Die in Art. 82 Abs. 1 Verordnung (EG) Nr. 987/2009 genannte Grenze ,wirtschaftliche und soziale Schwierigkeiten“" bedeutet, dass z.B. auf deutscher Seite nach $\S 54$ Abs. 4 SGB I die Vorschriften der $\S \S 850 \mathrm{ff}$. ZPO und die sich danach ergebenden Freibeträge zu beachten sind.

Die neuen Regelungen zur Beitreibung von Forderungen gehen deutlich über die Bestimmungen des vorhergehenden Verordnungsrechts zur Koordinierung der Systeme der sozialen Sicherheit hinaus, die lediglich die Beitreibung von Beitragsforderungen im Groben festlegte. 40 Aus Sicht der Praxis sind die nun getroffenen Änderungen grundsätzlich positiv zu bewerten, da den Mitgliedstaaten erstmalig ein ausdifferenziertes Instrument an die Hand gereicht wird, um dem vielfältigen Problem der Forderungsbeitreibung auch über Landesgrenzen hinweg wirksam begegnen zu können.

Erste praktische Umsetzungsmaßnahmen zwischen Deutschland und Polen wurden in dieser Fragestellung bereits vereinbart. So wurden im Rahmen der jüngsten deutschpolnischen Verbindungsstellenbesprechung im März 2011 bereits wesentliche Details für die praktische Verfahrensabwicklung abgestimmt und u.a. die grundsätzliche gegenseitige Anerkennung von Vollstreckungstiteln fixiert. Außerdem liegen nunmehr die entsprechenden Sprachversionen der insofern einschlägigen Structured Electronic Documents (SEDs) im XML-Format der Serie „R“ (Recovery = Rückforderung) vor. ${ }^{41}$

\section{Elektronischer Datenaustausch}

\section{1. Überblick}

Infolge des höheren Migrationsgeschehens nimmt u. a. für Arbeitgeber, Arbeitnehmer, Kommunen und insbesondere auch für die Sozialversicherungsträger die Bedeutung des elektronischen Datenaustausches weiter zu. Eine zeitgemäße, technisch unter-

40 Vgl. Art 92 Verordnung (EG) Nr. 1408/71 i. V. m. Art. 116 Verordnung (EG) Nr. 574/72.

41 Des Weiteren tagte eine Arbeitsgruppe der EU-Verwaltungskommission am 17.11.2011, um die Auslegung und Anwendung der Vorschriften zur Beitreibung zu konkretisieren. Die hieraus resultierenden Ergebnisse bleiben abzuwarten. 
stützte Bearbeitung der zunehmenden Fallzahlen ist aus Gründen der Effizienz sowie der Kundenorientierung (verkürzte Bearbeitungszeiten, Vermeidung und Reduzierung von Überzahlungen usw.) unabdingbar. Ein (weiterer) wesentlicher Schritt in diese Richtung ist im Rahmen des ab 01.05.2010 geltenden europäischen Koordinierungsrechts durch die Einführung eines elektronischen Datenaustausches, dem Electronic Exchange of Social Security Information (EESSI) vorgesehen. ${ }^{42}$

Parallel zu dieser EU-weiten Entwicklung finden allerdings bereits vielfache bilaterale elektronische Datenaustausche statt. Im Verhältnis zu Polen erfolgt dieser insbesondere mit dem seit 01.12.2011 im Einsatz befindlichen Europäischen OnlineAuskunftsverfahren (EOA-Polen). Ein maschineller Sterbedatenabgleich ist darüber hinaus bereits anvisiert. ${ }^{4}$

Damit kennzeichnen den Elektronischen Datenaustausch zwischen Deutschland und Polen derzeit drei Kernelemente:

- Electronic Exchange of Social Security Information (EESSI),

- Europäischem Online-Auskunftsverfahren mit Polen (EOA-Polen) und

- maschineller Sterbedatenabgleich Deutschland-Polen.

\section{Electronic Exchange of Social Security Information (EESSI)}

Kernstück der drei genannten Bereiche ist die gemeinschaftliche technische Infrastruktur EESSI. ${ }^{44}$ Für die Einführung von EESSI wurde den Mitgliedstaaten gem. Art. 95 Abs. 1 Verordnung (EG) Nr. 987/2009 eine Übergangszeit von 24 Kalendermonaten nach Inkrafttreten der Verordnung (EG) Nr. 987/2009, also bis zum 30.04.2012 eingeräumt. Eine angemessene Verlängerung dieser Übergangszeit kann allerdings von der Verwaltungskommission beschlossen werden, sofern sich die Bereitstellung des EESSI wesentlich verzögern sollte.

EESSI wird künftig der verpflichtende elektronische Austausch aller Formulare zwischen den beteiligten europäischen Trägern und gilt für alle Mitgliedstaaten und alle Sektoren, d.h. für die Rentenversicherung, die Krankenversicherung und die Unfallversicherung ebenso, wie für die Arbeitslosenversicherung und für Familienleistungen. ${ }^{45}$

42 Vgl. Art. 72 Buchst. d und Art 78 Verordnung (EG) Nr. 883/2004 i.V.m. Art 2 bis 5 Verordnung (EG) Nr. 987/2009.

43 In der Vergangenheit wurde der Datenaustausch z. B. über die Mitteilung von angepassten Rentenbeträgen per EU-Datensatz mit den polnischen Sozialversicherungsträgern praktiziert.

44 Vgl. www.ec.europa.eu/social (Koordinierung der Sozialversicherungssysteme der EU/EESSI). Die praktischen Verfahrensabläufe für den EESSI-Übergangszeitraum sind gem. Art. 95 Abs. 2 Verordnung (EG) Nr. 987/2009 im Beschluss der Verwaltungskommission Nr. E1 vom 12.06.2009, Abl. EU C 106 vom 24.04.2010 festgelegt worden.

45 www.ec.europa.eu/social (Koordinierung der Sozialversicherungssysteme der EU/Amtliche Dokumente). 
Kernelement des EESSI ist dabei der Austausch von Sozialversicherungsdaten unter Verwendung von Structured Electronic Documents (SEDs) für Anträge, Mitteilungen und Anfragen unter Nutzung von sTesta, d.h. einer von der europäischen Kommission mitgetragenen, datengeschützten Web-Anwendung sowie des WebIC als Anwenderoberfläche. Für die Anwender bei der Deutschen Rentenversicherung erfolgt die Anbindung an den entsprechenden Sachbearbeiterdialog, der auf WebIC nur im Hintergrund zugreift. Die Anwendungen werden damit bei der technischen Infrastruktur der Rentenversicherung berücksichtigt.

EESSI erfasst mit dem Austausch von Sozialversicherungsdaten, Mitteilungen, Anträgen und Anfragen bereits wesentliche Bereiche des praktischen Verwaltungsablaufs. Die Vorteile zeigen sich dabei in einer schnelleren Bearbeitung durch den Wegfall von Postzustellungen und durch die maschinelle Einspielung der Daten sowie in einer besser steuerbaren Organisation und Bearbeitung der einzelnen Geschäftsvorfälle. Allerdings erfasst EESSI keine sog. Portablen Dokumente (z.B. die Entsendebescheinigung A1), da diese nicht für den Austausch zwischen den Trägern vorgesehen sind, sondern den Berechtigten ausgehändigt werden. 46

Der Einsatz des elektronischen Datenaustausches war ursprünglich bereits zum 01.05.2012, d.h. nach Ablauf der Übergangszeit, vorgesehen. Nach Feststellungen der europäischen Verwaltungskommission kann jedoch der Zeitplan nicht eingehalten werden und damit ein vollständiger Datenaustausch zu diesem Zeitpunkt noch nicht erfolgen. Mit Sitzung vom 18./19.10.2011 hat daher die europäische Verwaltungskommission eine Verschiebung des Endes der Übergangszeit beschlossen. EESSI soll nun europaweit bis Ende Juni 2012 zur Verfügung stehen, um die technischen Anpassungen und den Einsatz auf nationaler Ebene bis April 2014 entsprechend vorbereiten zu können. ${ }^{47}$

\section{Europäisches Online-Auskunftsverfahren mit Polen (EOA-Polen)}

Das zweite Kernelement des elektronischen Datenaustausches mit Polen besteht im Europäischen Online-Auskunftsverfahren (EOA-Polen). In Umsetzung des Beschlusses Nr. 192 der Verwaltungskommission vom 29.10.2003 wird durch das Europäische Online-Auskunftsverfahren der Deutschen Rentenversicherung anderen europäischen Rentenversicherungsträgern die Einsicht der in den deutschen Versicherungskonten gespeicherten Versicherungszeiten und Rentenzahlbeträge ermöglicht.

46 Gemeinsame Arbeitsanweisungen, Art. 95 Verordnung (EG) 987/2009, Abschnitt R3.1 (abrufbar unter www.deutsche-rentenversicherung-berlin-regional.de).

47 Beschluss der Verwaltungskommission Nr. E3 vom 19.10.2011, Abl. EU C 12 vom 14.01.2012. 
Infobox 4: Abschluss des Europäischen Online-Auskunftsverfahrens mit Polen (EOA-Polen)

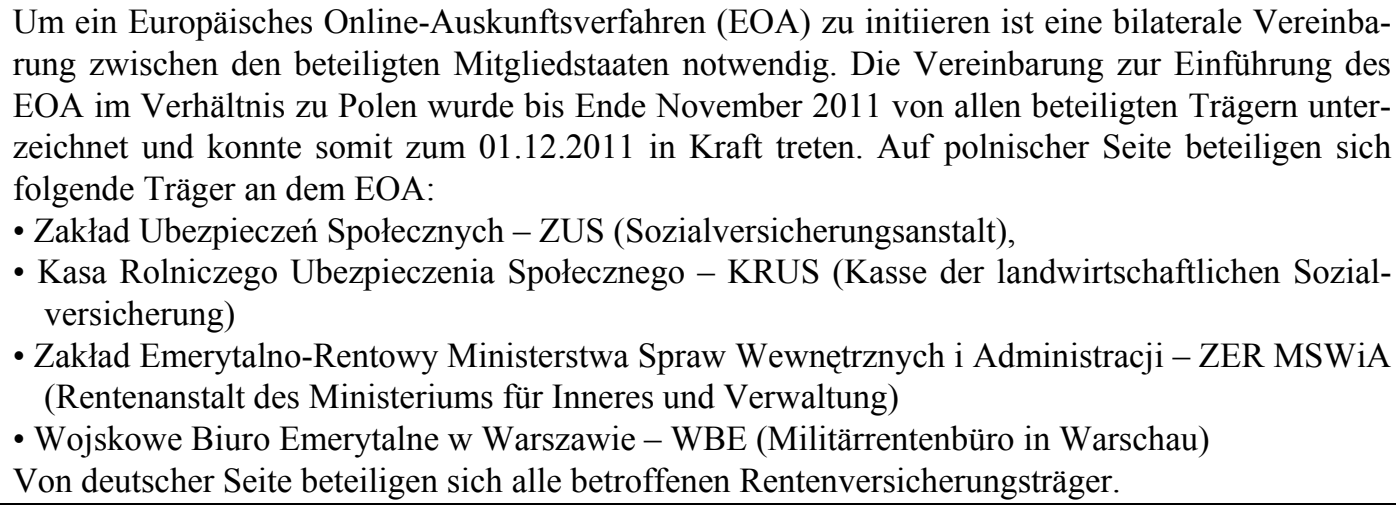

- Kasa Rolniczego Ubezpieczenia Społecznego - KRUS (Kasse der landwirtschaftlichen Sozialversicherung)

- Zakład Emerytalno-Rentowy Ministerstwa Spraw Wewnętrznych i Administracji - ZER MSWiA (Rentenanstalt des Ministeriums für Inneres und Verwaltung)

- Wojskowe Biuro Emerytalne w Warszawie - WBE (Militärrentenbüro in Warschau)

Von deutscher Seite beteiligen sich alle betroffenen Rentenversicherungsträger.

Durch den Einsatz des EOA-Polen wird der bis dato mit Polen praktizierte Schriftwechsel in Papierform zunehmend an Bedeutung verlieren. Stattdessen wird künftig ein Zugriff mittels EOA aus Polen auf die Daten in den deutschen Versicherungskonten ohne Einschaltung der deutschen Sachbearbeitung möglich sein. Im Gegenzug wird ein Zugriff mittels EOA aus Deutschland auf polnische Daten jedoch vorerst nur bedingt zu realisieren sein, da bei den einzelnen polnischen Rentenversicherungsträgern noch kein umfassender Datenbestand vorhanden ist.

Das EOA-Polen wird dabei auch nach Einführung von EESSI seine Bedeutung nicht einbüßen, da EESSI eine vergleichbare Funktion nicht vorsieht. Es wird deshalb (weiter) eine sinnvolle Ergänzung sein, da durch den unmittelbaren Zugriff die gewünschten Daten eingesehen werden können und damit eine schnellere Bearbeitung erreicht wird.

\section{Sterbedatenabgleich Deutschland-Polen}

Das dritte Element des elektronischen Datenaustausches mit Polen umfasst den maschinellen Sterbedatenabgleich. Dieser wird bereits zwischen verschiedenen Mitgliedstaaten und den zuständigen in- und ausländischen Rentenversicherungsträgern praktiziert (u.a. Deutschland mit Belgien, Italien, Luxemburg, Niederlande und Österreich). Ein solcher Abgleich stellt ebenfalls eine erhebliche Vereinfachung des Verwaltungsverfahrens dar, da Überzahlungen mit den Folgewirkungen wie Recherchearbeiten und Beitreibungen vermieden werden.

Ausgangspunkt der Überlegungen zur Verbesserung ist der Anspruch auf Rentenleistungen, der längstens bis zum Ablauf des Todesmonats des Berechtigten besteht. Damit keine Überzahlungen aufgrund verspäteter Zahlungseinstellung entstehen, muss der leistungsgewährende Träger rechtzeitig über den Tod eines Leistungsempfängers Kenntnis erlangen. Bei Inlandsrentenzahlungen in Deutschland wird dies seit 01.09.1984 durch die Verordnung zur Durchführung von regelmäßigen Datenübermittlungen der Meldebehörden oder sonstigen öffentlichen Stellen des Bundes (2. BMeld- 
DÜV) gewährleistet. ${ }^{48}$ Eine vergleichbare Verordnung mit Polen besteht nicht, weshalb vielfach Überzahlungen erfolgen, da der zahlende Rentenversicherungsträger erst verzögert von dem Versterben des Berechtigten in Polen erfährt.

Um diese Überzahlungen so gering wie möglich zu halten, wird bislang in Auslandsrentenfällen (im Verhältnis zu Ländern, mit denen noch kein maschineller Sterbedatenabgleich praktiziert wird) einmal jährlich durch die rentenzahlende Stelle eine Lebensbescheinigung mittels bundeseinheitlichem Vordruck „Lebensbescheinigung für Auslandszahlungen“ unter Bestätigung der Daten aus dem jeweiligen nationalen Bevölkerungsregister über den Berechtigten eingeholt. Dies erfolgt bisher auch mit Polen. Für die Einführung eines maschinellen Sterbedatenabgleichs wurden im Rahmen eines Treffens mit dem Renten Service der Deutschen Post AG im Juni 2011 die Möglichkeiten und Vorteile eines solchen Verfahrens mit den polnischen Trägern erörtert. Der Sterbedatenabgleich soll dabei unter Zugriff auf das polnische zentrale Melderegister (PESELRegister) unter grundsätzlicher Nutzung des europäischen sTesta-Netzes erfolgen. In einer Testphase mit der polnischen Seite soll dabei ein spezieller Server mit Zugriffsberechtigung und Datenfernübertragung mittels Secure File Transfer Protocol (SFTP, d.h. einer speziellen Anwendung zur Datenübertragung im geschützten Raum) zum Einsatz kommen.

Die weitere Realisierung hängt von der weiterhin ausstehenden Zustimmung einzelner polnischer Träger zur Testphase ab. Darüber hinaus werden die rechtlichen Voraussetzungen und die technische Machbarkeit eines maschinellen Sterbedatenabgleichs derzeit noch auf polnischer Seite geprüft. Die Direktion des polnischen PESELRegisters (zentrales Melderegister) hat zwischenzeitlich die grundsätzliche Bereitschaft zur Kooperation bestätigt. Aufgrund der weiteren noch offenen Fragen ist ein fester Termin für den Einsatz des elektronischen Sterbedatenabgleichs allerdings noch nicht festgelegt.

\section{E. Fazit}

Die Freizügigkeit in der Europäischen Union zeigt sich jeden Tag bei Reisen, bei der Arbeit, bei der Ausbildung und auch bei der Festlegung des Altersruhesitzes. Diese Entscheidungsfreiheiten basieren auf dem Grundrecht auf Freizügigkeit nach Art. 45 der Charta der Grundrechte der Europäischen Union sowie auf der Grundfreiheit der Arbeitnehmerfreizügigkeit nach Art. 45 ff. des Vertrags über die Arbeitsweise der Europäischen Union. Für den Bereich der gesetzlichen Rentenversicherung sind dabei die Verordnungen (EG bzw. EWG) zur Koordinierung der Systeme der sozialen Sicherheit von besonderer Bedeutung. Mit ihnen sollen Nachteile im Hinblick auf die Absicherung

$48 \S 5$ 2. BMeldDÜV. 
sozialer Risiken, die aus der Inanspruchnahme der Freizügigkeit entstehen könnten, vermieden bzw. reduziert werden.

In der Verwaltungspraxis zeigt sich hierbei eine stetige Zunahme der Nutzung dieser Freizügigkeit gerade zwischen Deutschland und Polen. Diese Entwicklung spiegelt sich im Hinblick auf die Sicherung im Alter in einer deutlich gestiegenen Anzahl an entsprechenden Versicherungskonten, einem höheren Beratungsbedarf und einer sprunghaft gestiegenen Anzahl an sog. Entsendebescheinigungen wider. Darüber hinaus ist eine deutliche Zunahme an Rentenzahlungen aufgrund von deutschen und polnischen Versicherungszeiten sowie eine höhere Anzahl an Leistungszahlungen von Deutschland nach Polen feststellbar. Damit nehmen für die Verwaltung Fragestellungen zur Erwerbstätigkeit ebenso wie zum Leistungsbezug weiter zu. Allerdings zeigt sich bei den Entwicklungen in der Erwerbsphase, wie auch beim Rentenbezug nicht nur verwaltungstechnischer (Stichworte sind Europäisches Online-Auskunftsverfahren mit Polen (EOAPolen)) und Electronic Exchange of Social Security Information (EESSI)), sondern auch politischer bzw. rechtlicher Handlungsbedarf. So ermöglichen die derzeitigen Regelungen zur Freizügigkeit im Zusammenspiel mit den deutschen sozialversicherungsrechtlichen Regelungen eine Umgehung von Sozialversicherungsbeitragszahlungen. Dies geht zu Lasten des Einzelnen in Form eines fehlenden Versicherungsschutzes und geringeren Vorsorgeleistungen, wie auch zu Lasten der Sozialen Sicherungssysteme in Form geringerer Einnahmen. Hier sind Maßnahmen angezeigt. Im Hinblick auf die Leistungsbezugsphase bleibt zunächst abzuwarten, wie das Vertragsverletzungsverfahren gegen Deutschland in der Frage zur Kürzung einer Rente bei Verlegung des Wohnsitzes von Deutschland nach Bulgarien ausgehen wird. Mögliche Konsequenzen für die vorliegend im Mittelpunkt stehenden Fragen im Verhältnis von Deutschland und Polen sind noch offen. 


\section{Die Freizügigkeit und die Realisierung der Regelungen im Bereich des Sozialschutzes zwischen Polen und Deutschland aus der Sicht der Sozialversicherungsanstalt/Abteilung in Oppeln}

\section{Bogdan Borecki}

A. Einführung und Entwicklung $\quad 257$

$\begin{array}{ll}\text { I. Entwicklung bis } 1990 & 257\end{array}$

II. Statistischer Hintergrund 259

III. Abschluss des neuen Sozialversicherungsabkommens 259

IV. Beitritt Polens zur EU 260

B. Anwendungsfragen $\quad 261$

I. Anwendbarkeit der rechtlichen Grundlagen 261

II. Folgen einer Rückkehr nach Polen 262

III. Weitere Fragen 263

1. Leistungsbewilligung $\quad 263$

2. Leistungsexport 264

3. Verschiedene Anspruchsvoraussetzungen 265

4. Sachleistungen 266

$\begin{array}{ll}\text { C. Schluss } & 266\end{array}$

\section{A. Einführung und Entwicklung}

\section{Entwicklung bis 1990}

Dieser Bericht ist ein Versuch, einen kleinen Ausschnitt der internationalen Zusammenarbeit im Bereich des Sozialschutzes darzustellen. Er betrifft die Aufgaben, die durch die Abteilung für Abwicklung internationaler Verträge der Sozialversicherungsanstalt in Oppeln im Rahmen der deutsch-polnischen Zusammenarbeit umgesetzt werden.

Eine der ersten Bestimmungen in diesem Bereich ist das Abkommen zwischen der Volksrepublik Polen und der DDR vom 13. Juli 1957 über die Zusammenarbeit auf dem Gebiet der Sozialpolitik. Das ist ein bilaterales Abkommen mit einem „territorialen“ Charakter. Auf der polnischen Seite betrifft es Arbeiter, die in Polen wohnen und in der Vergangenheit in der DDR gearbeitet haben. Der territoriale Charakter besteht darin, 